\title{
Imaging Mean Energy of X-ray Spectra through Intensity Variation in Radiographs with an Example Application to Beam Hardening Correction.
}

\author{
$\underline{\text { Andrew M. Kingston }}^{1,2,{ }^{*}}$, Wilfred K. Fullagar ${ }^{1}$, Glenn R. Myers ${ }^{1,2}$ and Adrian P. Sheppard ${ }^{1,2}$
}

1. Dept. of Applied Mathematics, RSPE, Australian National University, Canberra, ACT 2601, Australia.

2. CTLab: National Micro-CT Lab., Advanced Imaging Precinct, ANU, Canberra, ACT 2601, Australia.

* Corresponding author, andrew.kingston@anu.edu.au

X-ray detector response often scales with photon energy (bandgap energies are comparatively small), with quantum shot noise following a Poisson distribution. In computed tomography (CT) this information is predominantly used to develop maximum likelihood tomographic reconstruction algorithms. A different approach to the well-studied Poisson distribution can yield X-ray photon energy by combining a signal with its variance [1]. This spectral capability arises even with conventional polychromatic X-ray sources and cheap energy-integrating detectors. This opens new imaging modalities, with the capability for more quantitative imaging with fewer artefacts and the potential to discriminate materials that appear identical in conventional X-ray imaging. We present beam-hardening correction as an example application.

By taking multiple images (or radiographs) one can determine both the mean intensity, $I$, and the intensity variance, $\sigma^{2}$, per pixel. Consider monochromatic radiation with a mean of $N$ photons detected with energy $E$; we have $I=N E \Gamma$ where $\Gamma$ is a detector dependant parameter that encompasses phenomena such as luminescent conversion efficiency and electronic gain. Assuming a Poisson distribution, the standard deviation in number of photons is $\sqrt{N}$, with corresponding standard deviation in intensity $\sigma=\sqrt{N} E \Gamma$, and intensity variance $\sigma^{2}=N E^{2} \Gamma^{2}$. X-ray energy can be obtained from the ratio of variance and mean as $\sigma^{2} / I=E \Gamma$. In the polychromatic case, $I=\int S(E) \Gamma(E) d E$, where $S(E)$ is the energy weighted spectrum, i.e., $S(E)=E N(E)$, and thus $\sigma^{2}=\int S(E) E \Gamma^{2}(E) d E$. Observing that experimentally $\Gamma$ is constant, i.e., $\Gamma(E)=\gamma$, the ratio of variance and mean now yields the mean energy of the spectrum, $\langle E\rangle$, as follows:

$$
\frac{\sigma^{2}}{I}=\frac{\gamma^{2} \int S(E) \mathrm{E} d E}{\gamma \int S(E) d E}=\gamma\langle E\rangle
$$

Figure 1 gives experimental results that demonstrate $\Gamma$ is well approximated by a constant, and allow the calibration of our detector. For our W-bremsstrahlung source, three different accelerating voltages $\{60$, $90,120\} \mathrm{kV}$ and three different beam filters \{none, $2 \mathrm{~mm} \mathrm{Al}$, and $0.25 \mathrm{~mm} \mathrm{Cu}$ with $0.5 \mathrm{~mm} \mathrm{Al}$ \} the mean ratio of variance over intensity is plotted against the average energy of the spectra measured at the detector (assuming a CsI scintillator with a depth of $700 \mu \mathrm{m}$ ). We observe a linear trend with slope, $\gamma=0.00331$.

A sample composed of an $8 \mathrm{~mm} \mathrm{Al}$ rod and a $10 \mathrm{~mm}$ rod of $\mathrm{CaCO}_{3}$ is placed $40 \mathrm{~mm}$ from a Wbremsstrahlung source at $70 \mathrm{kV}$, filtered by $0.25 \mathrm{~mm} \mathrm{Al}$. A $360 \times 360 \mathrm{px}$ detector $785.5 \mathrm{~mm}$ from the source collected 360 mean \& variance radiographs. Each mean \& variance image is determined from 240 radiographs with $0.5 \mathrm{~s}$ exposure. The effects of beam-hardening $(\mathrm{BH})$ are evident in Fig. $2(\mathrm{~L})$ as cupping near the edges of rods and streaking between rods. The cause of these artefacts is that low energy photons have higher probability of absorption, so deep projections shift the transmitted spectrum to higher energies. We can now directly observe the spectral shift cf. the flatfield, $\mathrm{E}_{0} / \mathrm{E}$, as the ratio of $\sigma_{0}^{2} / I_{0}^{2}$ and $\sigma^{2} / I^{2}$ and compensate to avoid reconstruction artefacts. 

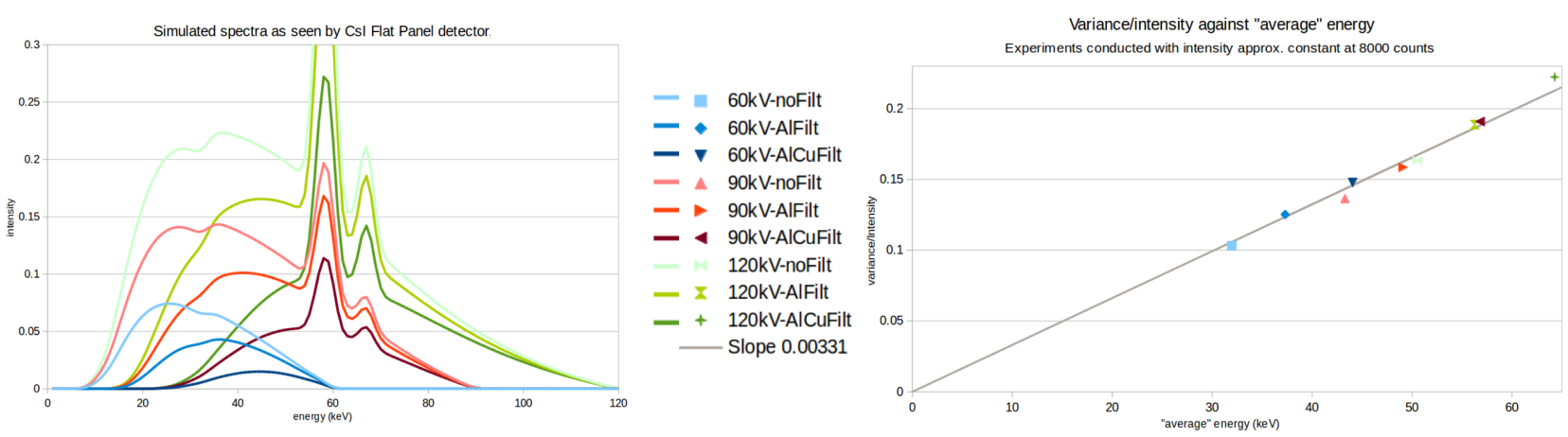

Figure 1: (L) Nine spectra generated for calibration, (R) Plot of $\sigma^{2} / I$ against $\langle E\rangle$; slope $=\gamma$.

A soft spectrum implies attenuation due to the photoelectric effect ( $\left.\propto E^{-3}[2]\right)$; Figure 2(C) shows the result of correcting $\mathrm{BH}$ by scaling the linearised projection images, $P$, by $\left(E_{0} / E\right)^{3}$. Qualitatively, the cupping, streaking, and contrast have all been significantly improved but the image is noisy. Quantitatively, the mean attenuation values are $\mathrm{Al}=2.1 \mathrm{~mm}^{-1}$ and Marble $=0.31 \mathrm{~mm}^{-1}$ which corresponds to attenuations at $35 \mathrm{keV}$ and $36 \mathrm{keV}$ respectively cf. mean X-ray energy of spectrum model at $38.4 \mathrm{keV}$. If correcting $\mathrm{BH}$ by linearisation [3] and using a power law model to map $P$ to $A P^{n}$, parameters $A$ and $n$ can be found as those minimising $\left\|\left(E_{0} / E\right)^{3} P-A P^{n}\right\|_{2}$. For the example presented here, $\mathrm{A}=1.39$ and $\mathrm{n}$ $=1.25$ are optimal. The result depicted in Fig. 2(R) has the qualitative improvements of previous method, without the additional noise; it also remains quantitative, i.e., $\mathrm{Al}=0.215 \mathrm{~mm}^{-1}$ and marble $=0.310 \mathrm{~mm}^{-1}$.
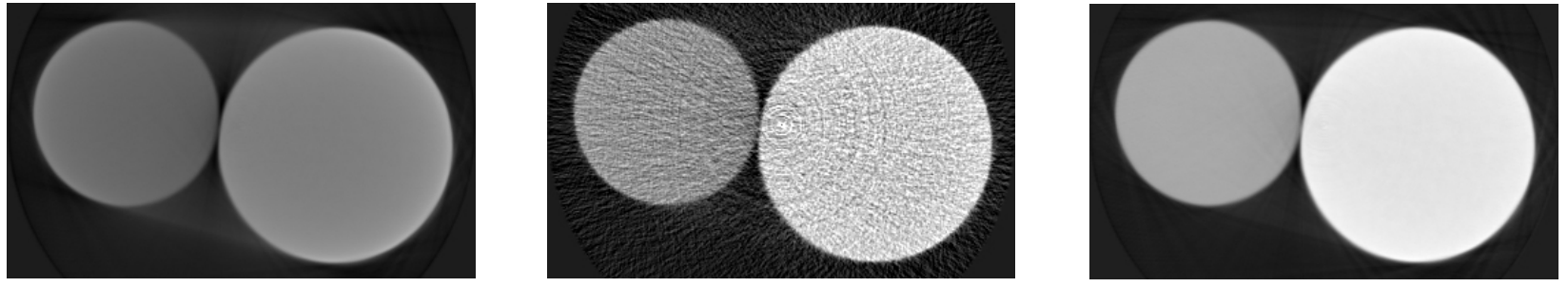

Figure 2: Tomographic reconstruction of $(\mathrm{L})$ mean data, $P,(\mathrm{C}) \mathrm{BH}$ correction of mean data as $P\left(E_{0} / E\right)^{3}$, (R) Linearisation BHC of mean by power law as $1.39 P^{1.25}$. Greyscale $[-0.05,0.35) \mathrm{mm}^{-1}$.

We have shown that average spectrum energy can be measured from intensity mean and variance using a conventional energy-integrating detector. A model for the energy weighted spectrum, $S(E)$, and a constant detector response with energy, $\Gamma(E)=\gamma$, are important requirements. This allows direct measurement of beam hardening which enables parameter-free correction techniques (or automated parameter determination); as demonstrated. This additional information could also be used in optimisation-based tomographic reconstruction such as maximum likelihood, and many other applications are still to be explored.

\section{References:}

[1] Fullagar et al., Acta Crystallographica Section B: Structural Science 73:4 (2017), p.675-695

[2] Alvarez, R.E. and Macovski, A., Physics in Medicine \& Biology 21:5 (1976), p.733

[3] Herman, G.T., Physics in Medicine \& Biology 24:1 (1979), p.81

[4] AMK, GRM and APS acknowledge the financial support of the Australian Research Council and Thermo Fisher Scientific through Linkage Project LP150101040. 